\title{
Retinal microvascular changes in white
} matter hyperintensities investigated by swept source optical coherence tomography angiography

Yuzhu Gao ${ }^{1+} \mathbb{0}$, William Robert Kwapong ${ }^{2 \dagger}$, Yifan Zhang ${ }^{1}$, , Yuying Yan ${ }^{2}$, Xurui Jin ${ }^{3}$, Yunhan Tao ${ }^{1}$, Hanyue Xu', Bo $\mathrm{Wu}^{2^{*}}$ and Ming Zhang ${ }^{1 *}$ (1)

\begin{abstract}
Backgro: To assess the microvascular changes in the macular region and the foveal avascular zone (FAZ) area in participants with white matter hyperintensities (WMHs) using swept source optical coherence tomography angiography (SS OCTA).

Methods: This cross-sectional study included a total of $23 \mathrm{WMH}$ participants (45 eyes) and 20 age-matched healthy participants (40 eyes). SS OCTA (VG200; SVision Imaging, Ltd., Luoyang, China) was used to assess the retinal vessel density (VD) and the FAZ area. VD was measured in the superficial vascular plexus (SVP), intermediate capillary plexus (ICP) and deep capillary plexus (DCP) within a $6 \times 6-\mathrm{mm}$ scan centred on the macula using a 5-mm Macula circle. The FAZ area was automatically measured on the inner retina layer within a $3 \times 3-\mathrm{mm}$ scan in the macular region.

Results: There was no significant difference in VD in the SVP between the two groups. However, VD in both the ICP and DCP was significantly decreased in WMH participants $(P=0.028, P=0.016)$. The FAZ area was significantly enlarged in WMH participants $(P=0.030)$. The signal quality was significantly lower in WMH participants $(P<0.001)$.

Conclusions: This study suggested that WMH participants have retinal microvascular and foveal avascular zone area changes compared with healthy controls. Further longitudinal studies with larger sample sizes are warranted to identify the value of our findings in the early evaluation of WMHs.
\end{abstract}

Keywords: White matter hyperintensities, Retina, Foveal avascular zone, Swept source optical coherence tomography angiography, Microvascular

*Correspondence: dr.bowu@hotmail.com; mingzhangscu@163.com †Yuzhu Gao and William Robert Kwapong co-first authors: these authors contributed equally to this project.

1 Department of Ophthalmology, West China Hospital, Sichuan University, No.37 Guoxue Lane, Chengdu Zip code: 610041, Sichuan Province, China ${ }^{2}$ Department of Neurology, West China Hospital, Sichuan University, No.37 Guoxue Lane, Chengdu Zip code: 610041, Sichuan Province, China Full list of author information is available at the end of the article

\section{Background}

Alzheimer's disease (AD) is the most common cause of dementia and has been reported to affect millions of elderly individuals worldwide [1, 2]. A loss of neurons, atrophy of the brain and deposition of amyloid plaques are normally associated with the pathophysiologic process of $\mathrm{AD}$ [3]. Nonetheless, the classic symptoms of AD, progressive memory loss and changes in behaviour, are recognized as occurring or manifesting after considerable, irreversible neuroaxonal loss in the brain $[4,5]$. Thus, original author(s) and the source, provide a link to the Creative Commons licence, and indicate if changes were made. The images or other third party material in this article are included in the article's Creative Commons licence, unless indicated otherwise in a credit line to the material. If material is not included in the article's Creative Commons licence and your intended use is not permitted by statutory regulation or exceeds the permitted use, you will need to obtain permission directly from the copyright holder. To view a copy of this licence, visit http://creativecommons.org/licenses/by/4.0/. The Creative Commons Public Domain Dedication waiver (http://creativeco mmons.org/publicdomain/zero/1.0/) applies to the data made available in this article, unless otherwise stated in a credit line to the data. 
clinicians have suggested that the early phase or preclinical phase of $\mathrm{AD}$ may be the new route to help develop timely and effective diagnostic approaches to help slow the progression of the disease. Furthermore, with such tools, early-stage targeted intervention could be conducted to prevent $\mathrm{AD}$.

White matter hyperintensities (WMHs), as measured on magnetic resonance imaging (MRI), are normally found in the ageing population and are characterized as hyperintensities on T2-weighted or flair-attenuated inversion recovery (FLAIR) images. Reports have shown that WMHs are found in over $50 \%$ of older adults [5, 6 ] and are associated with the development of $\mathrm{AD}$ and dementia $[7,8]$. Although the pathogenesis and clinical relevance of WMHs are still vague, recent studies have suggested that WMHs are marked by microvascular dysfunction in the cerebral microcirculation [9], and some pathological studies have suggested that cerebral small vessel changes occur in WMH participants. In addition, some cerebral imaging studies found significant neuronal loss in WMH participants [10, 11]. However, whether microvascular dysfunction contributes to neuronal loss in the brain is unclear. It is vital to be able to detect the association between them.

Optical coherence tomography (OCT) is a well-tolerated, noninvasive imaging modality that provides highresolution images of the retina and has been widely applied in numerous fields, especially ophthalmic imaging $[12,13]$. Recent reports have suggested that retinal microcirculation could be a useful tool to evaluate cerebral microcirculation [14]. Previous retinal imaging reports have found venous narrowing and reduced blood flow in WMH participants compared with healthy controls [15-17]. A study using optical coherence tomographic angiography (OCTA) evaluated the retinal microvasculature in WMH participants and suggested that decreased deep capillary density was associated with WMHs [18]. However, detailed information on the capillary plexus of the macula and the foveal avascular zone (FAZ), an important indicator of $\mathrm{AD}$, was not studied in previous reports. Swept source optical coherence tomographic angiography (SS OCTA) is a new and improved noninvasive retinal imaging modality that can help to visualize and evaluate the in-depth and deepest capillaries of the macula. Hence, SS OCTA may enable quick, inexpensive, and noninvasive screening of WMHs.

We hypothesized that the changes in the retina may reflect similar changes in the brain in WMH participants. Accordingly, we used SS OCTA to assess the macular vessel density (VD) and FAZ area in WMH participants and healthy control participants. Additionally, we also investigated whether the SS OCTA tool has the potential to characterize the retinal microvasculature in WMHs.

\section{Materials and methods}

\section{Study participants}

Participants with confirmed WMHs and age-matched healthy participants were recruited in this cross-sectional study. All the WMH participants were dementia- and stroke-free but had white matter lesions on MRI (Fazekas score greater than 1 but less than or equal to 3 ) and in the subcortical region of the brain. The inclusion criteria for the WMH participants were as follows: 1) aged over 50 years; 2) could cooperate during MRI and retinal imaging; and 3) Chinese. The exclusion criteria were as follows: 1) eyes with known ocular diseases such as glaucoma and age-related macular degeneration; 2) a refractive error of less than -6.0 dioptres and prior intraocular surgery; and 3) systemic disease that might affect eyes such as systemic lupus erythematosus and diabetes mellitus.

All participants underwent standard ophthalmic examination, including best-corrected visual acuity (BCVA) testing using the Snellen chart, slit-lamp biomicroscopy, intraocular pressure (IOP) measurement, and dilated fundus examination. Moreover, for WMH participants, Fazekas scores were obtained and MRI was performed for evaluation.

This study was approved by the Institutional Review Board for Human Research at West China Hospital, China, and conducted in compliance with the Declaration of Helsinki. All enrolled participants provided written informed consent.

\section{Optical coherence tomography angiography imaging and image processing}

All participants were imaged with SS OCTA with a scanning speed of 200,000 A scan per second, widefield angle of 56 degrees, and an imaging depth of $2.7 \mathrm{~mm}$ in tissue at $1050 \mathrm{~nm}$ (VG200; SVision Imaging, Ltd., Luoyang, China). Both $3 \times 3$ and $6 \times 6-\mathrm{mm}$ scans were performed in the centre of the macula three times for each participant, which consisted of 512 horizontal A lines at 512 vertical locations with four repeated scans in each fixed location, resulting in a sampling spacing of $12 \mu \mathrm{m}$.

The presence of blood vessels is directly indicated by angiography signals. An algorithm is designed to separate the foreground (blood vessel) pixels from background (non-vessel tissue) pixels by properly segmenting the image from the perspective of angiography signal strength. The VD is defined as a metric used to describe the percentage of area occupied by blood vessels in a two-dimensional retina projection image. The projection image is acquired by projecting threedimensional angiography volume data onto a twodimensional imaging plane, which is also called an en 
face image. As described, then the ratio of blood vessel pixels as a percentage of all pixels is computed within a pre-defined window size.

OCTA images selected with good scan quality (more than $7 / 10$ signal strength as defined by the manufacturer) and without severe motion artefacts were included. Retinal layers were segmented using a validated semiautomated segmentation algorithm and manually calibrated by two experienced ophthalmologists for accuracy. The superficial vascular plexus (SVP), the intermediate capillary plexus (ICP) and the deep capillary plexus (DCP) were automatically segmented by the software of the device. The SVP slab was segmented as the inner $80 \%$ of the ganglion cell complex (GCC) (defined as the nerve fibre layer $(\mathrm{NFL})+$ ganglion cell layer $(\mathrm{GCL})+$ inner plexiform layer (IPL)), excluding the NFL. The intermediate capillary plexus (ICP) was generated from the outer $20 \%$ of the GCC to the inner $50 \%$ of the inner nuclear layer (INL), the deep capillary plexus (DCP) was generated by a slab extending from the outer $50 \%$ of the INL to $25 \mu \mathrm{m}$ beneath the interface INL/outer plexiform layer (OPL), and the inner retina extended from $5 \mu \mathrm{m}$ above the interface ILM to $25 \mu \mathrm{m}$ beneath the interface INL/ OPL (Fig. 1).

The VD was calculated according to the 5-mm Macularing circle on the SVP, ICP and DCP centred on the macula from a $6 \times 6-\mathrm{mm}$ scan and assessed by the mean value of the 9 regions (Fig. 2). The FAZ area was automatically measured based on en face images of the inner retina layer from a $3 \times 3$-mm scan (Fig. 3).

\section{Statistical analysis}

Baseline characteristics are presented as the mean (continuous variables) or frequency distribution (categorical variables). The Shapiro-Wilk test was used to test the normality of the data. Generalized estimating equation (GEE) models were used to compare the differences in microvascular density while adjusting for risk factors and inter-eye dependencies. A $P$ value less than 0.05 was considered statistically significant throughout the study. Statistical analysis of collected data was performed using IBM SPSS Statistics version 23 (SPSS Inc., Chicago, IL, USA).

\section{Results}

A total of 23 WMH participants and 23 age-matched healthy control participants were recruited. Three healthy control participants were excluded owing to unpredictable drusen during the checking procedure. Thus, 45 eyes from $23 \mathrm{WMH}$ participants and 40 eyes from 20 healthy control (HC) participants were enrolled in imaging processing. Baseline characteristics are shown in Table 1. In all $23 \mathrm{WMH}$ participants, these white matter lesions were in the subcortical portion of the brain. The mean age of the WMH participants was $59.14 \pm 5.75$ years, and that of the healthy control participants was $56.37 \pm 3.55$ years.

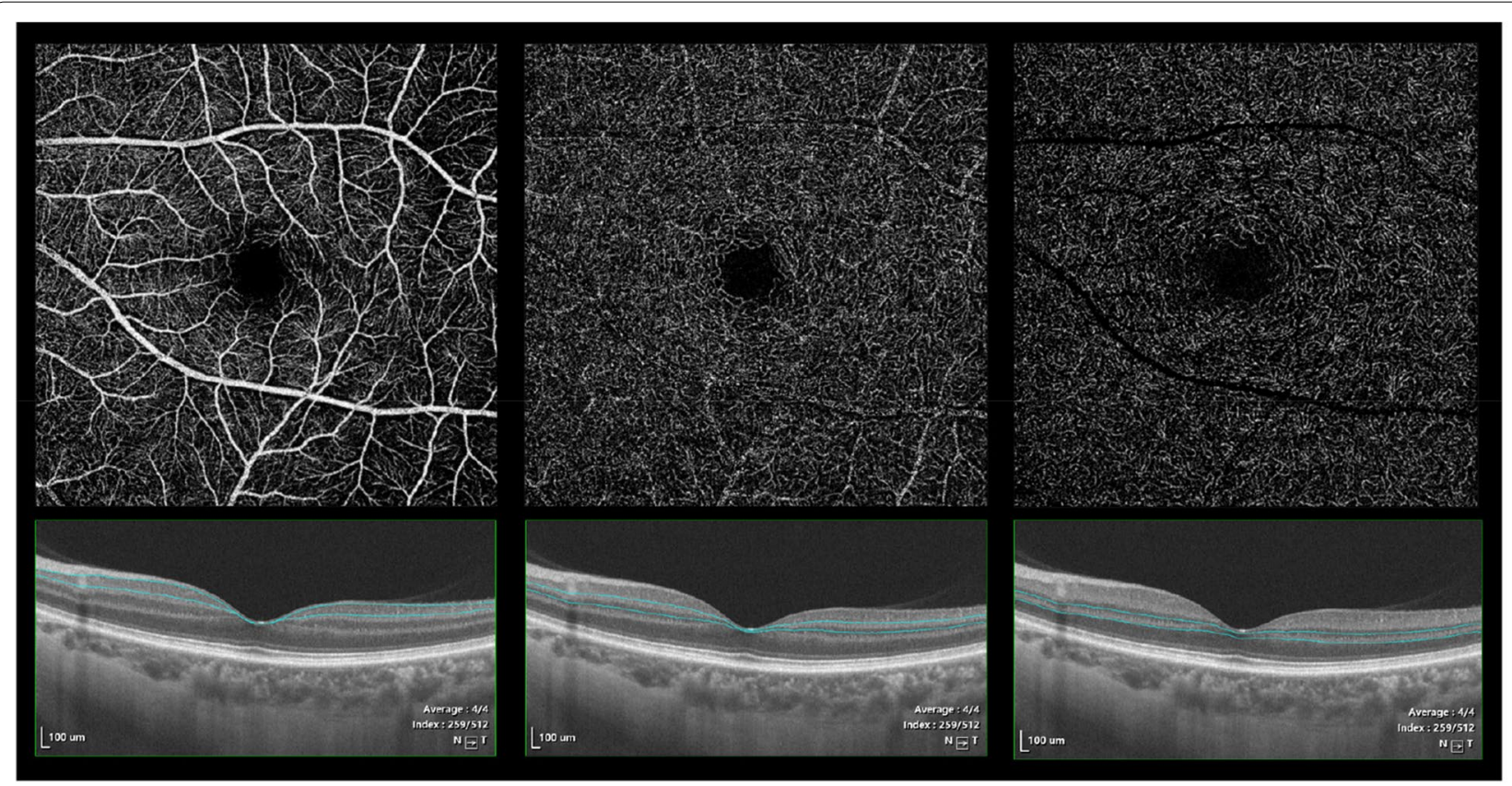

Fig. 1 En face SS OCTA images of three layers participates with corresponding segmenting slabs: A superficial vascular layer $\mathbf{B}$ intermediate capillary layer $\mathbf{C}$ deep capillary layer (from a WMH participant) 


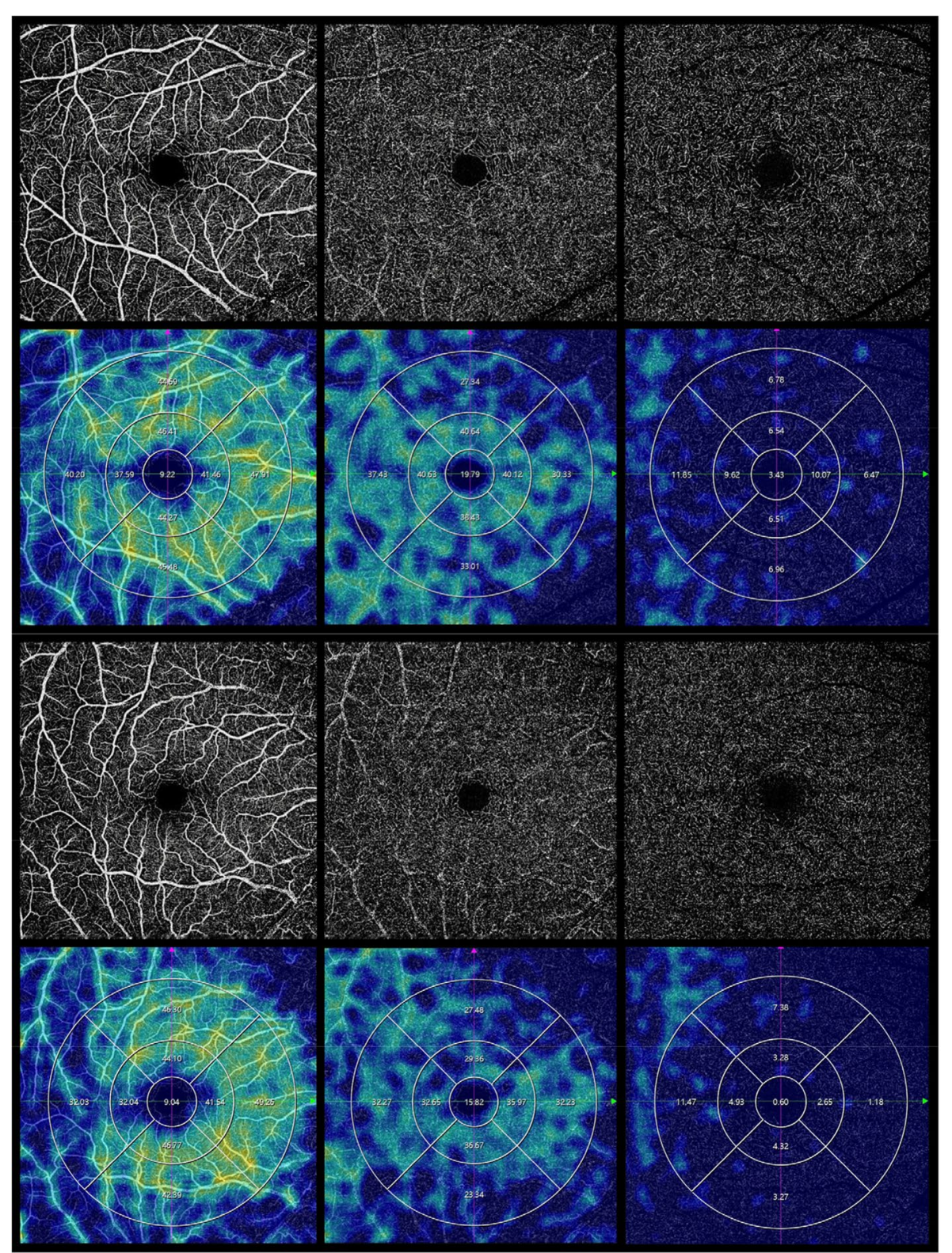

Fig. 2 Representative SS OCTA $6 \times 6$-mm image of vessel density of superficial vascular layer, intermediate capillary layer and deep capillary layer centred on macula, the vessel density decreased significantly in the WMH participant: a from a healthy control participant $\mathbf{b}$ from a WMH participant

There were no significant differences between groups in sex (with WMHs, 15 males and 8 females; 9 males and 11 females in healthy controls), IOP (with WMHs, $13.74 \pm 1.03 ; 14.09 \pm 2.13$ of healthy controls) or axial length (with WMHs, 22.94 $\pm 9.01 ; 23.03 \pm 7.20$ of healthy controls). The average LogMAR visual acuity was $0.13 \pm 0.16$ in the $\mathrm{WMH}$ group and $-0.01 \pm 0.12$ in the healthy control group $(P<0.001)$. The mean Montreal Cognitive Assessment (MoCA) score was 25.14 \pm 1.29 , and the mean Fazekas score was $2.2 \pm 0.45$ in the WMH group.

Quantitative measurements of VD in the SVP, ICP and DCP and FAZ area are shown in Table 2. Comparing the WMH group to the healthy control group, there was no significant difference in VD in the SVP $(P=0.521)$. However, the WMH group had significantly decreased VD in the ICP $(P=0.028)$ and DCP $(P=0.016)$ in the $5-\mathrm{mm}$ Macula circle compared to 


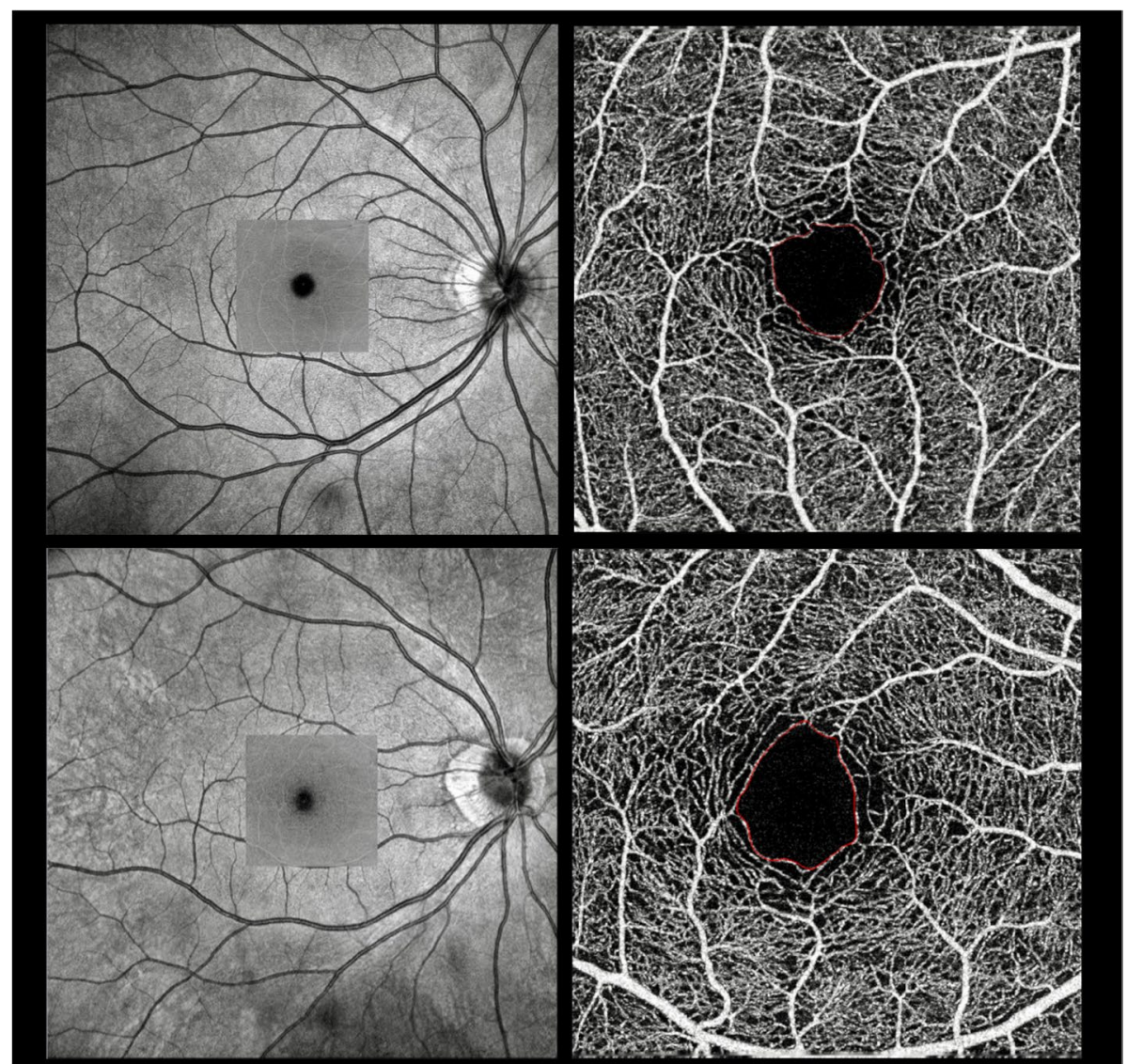

Fig. 3 Representative SS OCTA $3 \times 3-m m$ images of inner retina layer and delineation of the FAZ: $\mathbf{a}-\mathbf{b}$ from a Healthy control participant c-d from a WMH participant

Table 1 Baseline Characteristics

\begin{tabular}{lllr}
\hline & WMH & HC & P value \\
\hline Number & 23 & 20 & \\
Number of eyes & 45 & 40 & \\
Gender (M:F) & $15: 8$ & $9: 11$ & 0.498 \\
Age, years & $59.14 \pm 5.75$ & $56.37 \pm 3.55$ & 0.068 \\
IOP, mmHg & $13.74 \pm 1.03$ & $14.09 \pm 2.13$ & 0.396 \\
Axial length, mm & $22.94 \pm 9.01$ & $23.03 \pm 7.20$ & 0.821 \\
VA, LogMAR & $0.13 \pm 0.16$ & $-0.01 \pm 0.12$ & $<0.001$ \\
Hypertension, number & 6 & 0 & \\
MoCA score & $25.14 \pm 1.29$ & & \\
Mean Fazekas score & $2.2 \pm 0.45$ & & \\
Location of WMHs & & & \\
$\quad$ Subcortical, $\mathrm{n}$ & 23 & & \\
\hline
\end{tabular}

Abbreviations: WMH White Matter Hyperintensities, HC Healthy control, IOP Intraocular Pressure, VA Visual Acuity, MoCA Montreal Cognitive Assessment
Table 2 Comparison of vessel density in three layers and the foveal avascular zone area in inner retina

\begin{tabular}{llll}
\hline & WMH & HC & P value \\
\hline SVP, \% & $39.31 \pm 3.94$ & $40.13 \pm 5.69$ & 0.521 \\
ICP, \% & $31.34 \pm 4.79$ & $34.12 \pm 4.96$ & 0.028 \\
DCP, \% & $9.08 \pm 4.10$ & $10.82 \pm 3.24$ & 0.016 \\
FAZ, mm ${ }^{2}$ & $0.40 \pm 0.12$ & $0.31 \pm 0.08$ & 0.030 \\
Signal quality & $7.93 \pm 0.65$ & $9.55 \pm 0.50$ & $<0.001$ \\
\hline
\end{tabular}

Adjusted for age, gender, signal quality, hypertension, axial length and eyes Abbreviations: WMH White Matter Hyperintensities, HC Healthy control, SVP superficial vascular plexus, ICP intermediate capillary plexus, $D C P$ deep capillary plexus, FAZ foveal avascular zone area

the healthy control group. There was also a significant difference in FAZ area in the inner retina between the WMH and healthy control groups $(P=0.030)$. Moreover, the signal quality was significantly higher in the healthy control group than in the WMH group $(P<0.001)$. 


\section{Discussion}

In this study, we used SS OCTA to investigate the retinal microvasculature and the FAZ area in WMHs and healthy control participants. Our results showed significantly decreased VD in both the ICP and the DCP and an enlarged FAZ area in WMH participants compared with age-matched healthy control participants. These differences suggested that the FAZ area and the VD in the ICP and DCP may provide an earlier marker in screening for WMHs and the preclinical phase of AD.

Compared with healthy control participants, we found significantly decreased VD in both the ICP and the DCP of the retina in WMH participants. Peng and colleagues [18] compared the microvascular changes in WMH participants with different degrees of lesions and indicated that microvascular impairment was associated with the disease cascade in WMHs. Mutlu and colleagues [19] have also reported an association between retinal neurodegeneration and cerebral atrophy, indicating the role retinal OCT plays in providing information on neurodegeneration in the brain.

However, the mechanism of decreased microcirculation in WMHs is still unknown and needs further study. Previous studies have suggested that the changes in the retina reflect the similar pathological changes occurring in the brain [20], since the retina shares many similarities with the brain, including embryological origin, microvasculature and neuronal projections [21]. It is known that retinal vascular networks are composed of several layers that differ in location, composition and function. The SVP includes flow from the ILM to the middle of the IPL and mainly supplies the ganglion cell layer. It is a network and contains both large and small vessels [22]. The deep vascular plexus (DVP) has two subcomponents: the ICP, containing a slab extending from the IPL to the INL; and the DCP, extending from the INL to the OPL. The ICP and DCP are mainly composed of smaller vessels and capillaries [22]. Since the SVP mainly contains larger vessels and the DVP (ICP and DCP) is related to the function of capillary microcirculation, in our study, the significant decreases in the ICP and DCP may indicate the early appearance of microcirculation dysfunction in the retina in WMH participants. Thus, as a previous report suggested that WMHs may originate from chronic small vessel ischaemia [23], our study showed that what is seen in the retinal microcirculation may be a reflection of the changes in cerebral microcirculation. Additionally, morphologic substrate, induced by impaired diffusion, was found through thickened vessel walls in WMH participants [24]. Since those changes likely start with smaller vessels, it is of great possibility that those substrates may be present in the small vessels of the ICP and DCP, which may result in early vessel dropout and microvascular dysfunction.

Another possible explanation for decreased VD in the ICP and DCP may be alterations in oxygen. Previous retinal oxygen measurement experiments in animal models have demonstrated that for the inner retina, the dominant oxygen consumers may be located in the plexiform layers (IPL and OPL), probably in mitochondria-rich synapses $[25,26]$, indicating the need for a highly oxygenated blood supply. Notably, according to Hagag and colleagues [27], in healthy subjects, when compared with other capillary layers of retina, the plexiform layers may maximally constrict under the exposure to hyperoxia, which indicated that the plexiform layers may be influenced greatly under the hypoxic status. Hence, the impaired VD in the ICP and DCP may also be a reflection of the mitochondrial dysfunction and high oxygen consumption found in the brains with WMHs.

We found a significant enlargement of the FAZ area in WMH participants, which has rarely been reported in previous studies. The FAZ refers to the capillaryfree area centred on the macula. In this study, the FAZ area was automatically measured in the inner retina, which extends from $5 \mu \mathrm{m}$ above the interface ILM to $25 \mu \mathrm{m}$ beneath the interface INL/OPL. Quantifying FAZ parameters has been a useful tool in evaluating macular microcirulation [28]. Since a prior study showed a significant age effect on the FAZ area [29], we selected age-matched participants as controls. The mechanism underlying the enlarged FAZ area in WMH participants remains unknown, but microcirculation dysfunction has been reported to be a cause. Another possible explanation may be neurodegenerative changes. Partial loss of myelin, axons, and oligodendroglion cells in the brain is a common finding seen in WMH participants. The enlargement of the FAZ area in the inner retina may be secondary to both the impairment of neurons and glia in the retina and their effects on the decrease in flow density. Third, perivascular tissue changes, considered the prevailing morphologic substrate [30], may also be a potential explanation. According to $\mathrm{Ma} \mathrm{KC}$ and colleagues [31], perivascular oedema in the retina, due to intermittent disruption of the blood-brain barrier, could also lead to damage in VD, which results in enlargement of the FAZ area. Hence, the enlarged FAZ area may indicate the impairment of microcirculation in the brain.

Previous studies demonstrated that retinal microvascular density might be associated with axial length [32], sex [33] and refractive errors [34]. However, some remain disputable. Wen and colleagues [32] found that the axial length was negatively associated with superficial parafoveal vessel density but not correlated with VD around the optic nerve head and the FAZ area, while some previous 
studies indicated that the axial length was negatively correlated with the FAZ area [35]. Milani and colleagues [34] reported that eyes with high myopia were negatively related to superficial VD and positively associated with blood flow of the outer retina. Hence, we adjusted for these parameters during the evaluation.

We acknowledge several limitations in this study. The first is the limited sample size; however, we have used. Second, limited by our study design, we were not able to determine whether these results are related to individual differences. Therefore, we could not be able to determine whether our findings could be translatable to all WMH individuals. However, we designed strict recruitment and made adjustments for multiple comparisons. Further studies with larger sample sizes and longer follow-ups are needed. Third, all the participants were Chinese, which may make our findings hard to generalize to other ethnic groups. The fourth limitation is that we did not measure the microstructural volume of WMH participants. Moreover, most of our WMH participants were in the early stage of the disease; hence, we did not group people according to the severity of disease. Further studies with more detailed neuroimaging data and patients in different disease stages are warranted to enhance our findings and provide in-depth meaning.

There are also some strengths in our study. First, we used SS OCTA for evaluation. The unprecedented scanning speed, imaging depth and sensitivity of SS OCTA improved the accuracy of images. Second, we detected both VD and the FAZ area in WMH participants, which has rarely been reported before and may provide more possible imaging targets for the early diagnosis of WMHs.

\section{Conclusions}

In conclusion, our study showed that SS OCTA may be a new, quick, noninvasive, well-tolerated and inexpensive tool for screening WMH participants based on decreased VD and enlarged FAZ areas. Further longitudinal studies with larger samples are warranted to determine whether our findings have potential meanings in the early detection of WMHs; hence, these participants could be able to receive early care and treatment.

\footnotetext{
Abbreviations

AD: Alzheimer's disease; WMHs: White matter hyperintensities; MRI: Magnetic Resonance Imaging; FLAIR: Flair-attenuated inversion recovery; OCT: Optical coherence tomography; OCTA: Optical coherence tomographic angiography; FAZ: Foveal avascular zone; SS OCTA: Swept source optical coherence tomographic angiography; VD: Vessel density; IOP: Intraocular pressure; SVP: Superficial vascular plexus; ICP: Intermediate capillary plexus; DCP: Deep capillary plexus; GCC: Ganglion cell complex; NFL: Nerve fibre layer; GCL: Ganglion cell layer; IPL: Inner plexiform layer; INL: Inner nuclear layer; GEE: Generalized estimating eq.; HC: Healthy control; VA: Visual Acuity; MoCA: Montreal Cognitive Assessment.
}

Acknowledgements

Not applicable.

\section{Authors' contributions}

MZ, BW, YZG and WRK designed the study; YZG, WRK, YFZ, YYY, YHT and HYX collected data. YZG managed data and wrote the manuscript; WRK analyzed data; $M Z$ and $X R J$ reviewed the manuscript. All authors read and approved the final manuscript.

\section{Funding}

The authors declare that they have no funding.

\section{Availability of data and materials}

The datasets used and/or analyzed during the current study available from the corresponding author on reasonable request.

\section{Declarations}

\section{Ethics approval and consent to participate}

This study was approved by the Institutional Review Board for Human Research at West China Hospital, China and conducted in compliance with Declaration of Helsinki. All enrolled participants provided written informed consent.

\section{Consent for publication}

The written informed consent for publication was obtained from the participant.

\section{Competing interests}

The authors declare that they have no competing interests.

\section{Author details}

${ }^{1}$ Department of Ophthalmology, West China Hospital, Sichuan University, No.37 Guoxue Lane, Chengdu Zip code: 610041, Sichuan Province, China. ${ }^{2}$ Department of Neurology, West China Hospital, Sichuan University, No.37 Guoxue Lane, Chengdu Zip code: 610041, Sichuan Province, China. ${ }^{3}$ Duke Global Health Institute, Duke University, Durham, NC, USA.

Received: 28 December 2020 Accepted: 8 October 2021

Published online: 15 February 2022

\section{References}

1. Scott JA, Braskie MN, Tosun D, et al. Cerebral amyloid and hypertension are independently associated with white matter lesions in elderly. Front Aging Neurosci. 2015;7:221.

2. Fratiglioni L, De Ronchi D, Agüero-Torres H. Worldwide prevalence and incidence of dementia. Drugs Aging. 1999;15:365-75.

3. Morris ZN. Alzheimer's Disease in The Neuropathology Dementia. Cambridge: Univ Press UK; 2004. p. 161-206.

4. Uhlmann R, Larson E, Koepsell T, Rees T, Duckert L. Visual impairment and cognitive dysfunction in Alzheimer's disease. J Gen Intern Med. 1991;6:126-32.

5. Javaid F, Brenton J, Guo L, Cordeior M. Visual and Ocular Manifestations of Alzheimer's Disease and Their Use as Biomarkers for Diagnosis and Progression. Front Neurol. 2016:2016(7):1-11.

6. Breteler MM, van Swieten JC, Bots ML, et al. Cerebral white matter lesions, vascular risk factors, and cognitive function in a population-based study: the Rotterdam study. Neurology. 1994;44(7):1246-52.

7. Lau AY, Mok V, Lee J, et al. Retinal image analytics detects white matter hyperintensities in healthy adults. Ann Clin TransI Neurol. 2019;6(1):98-105

8. Seneviratne U, Chong W, Billimoria PH. Brain white matter hyperintensities in migraine: clinical and radiological correlates. Clin Neurol Neurosurg. 2013;115(7):1040-3. https://doi.org/10.1016/..clineuro.2012.10.033.

9. Pantoni L, Garcia J. Pathogenesis of leukoaraiosis: a review. Stroke. 1997;28(3):652-9. 
10. Chang YL, Yen YS, Chen TF, Yan SH, Tseng W. Clinical dementia rating scale detects white matter changes in older adults at risk for Alzheimer's disease. J Alzheimers Dis. 2016;50(2):411-23.

11. Zhang Y, Zhang GY, Zhang ZE, He AQ, Gan J, Zhenguo L. White matter hyperintensities: a marker for apathy in Parkinson's disease without dementia? Ann Clin Transl Neurol. 2020;7(9):1692-1701.

12. Dai $Y, Z$ Zhou H, Chu Z, et al. Microvascular changes in the choriocapillaris of diabetic patients without retinopathy investigated by swept-source OCT angiography. Investig Ophthalmol Vis Sci. 2020;61(3):1-8. https://doi. org/10.1167/iovs.61.3.50.

13. Yang J, Wang E, Yuan M, Chen Y. Three-dimensional choroidal vascularity index in acute central serous chorioretinopathy using swept-source optical coherence tomography. Graefes Arch Clin Exp Ophthalmol. 2020;258(2):241-7. https://doi.org/10.1007/s00417-019-04524-7.

14. London A, Benhar I, Schwartz M. The retina as a window to the brain-from eye research to CNS disorders. Nat Rev Neurol. 2013;9(1):44-53.

15. I kram MK, De Jong FJ, Van Dijk EJ, et al. Retinal vessel diameters and cerebral small vessel disease: the Rotterdam scan study. Brain. 2006;129(Pt 1):182-8.

16. Hilal S, Ong YT, Cheung CY, et al. Microvascular network alterations in retina of subjects with cerebral small vessel disease. Neurosci Lett. 2014;577:95-100.

17. Liew G, Baker ML, Wong TY, et al. Differing associations of white matter lesions and lacunar infarction with retinal microvascular signs. Int J Stroke. 2014:9(7):921-5.

18. Peng C, Kwapong WR, Xu S, et al. Structural and microvascular changes in the macular are associated with severity of white matter lesions. Front Neurol. 2020;11:1-8. https://doi.org/10.3389/fneur.2020.00521.

19. Mutlu U, Bonnemaijer PWM, Ikram MA, et al. Retinal neurodegeneration and brain MRI markers: the Rotterdam study. Neurobiol Aging. 2017:60:183-91. https://doi.org/10.1016/j.neurobiolaging.2017.09.003.

20. Patton N, Aslam T, MacGillivray T, Pattie A, Deary IJ, Dhillon B. Retinal vascular image analysis as a potential screening tool for cerebrovascular disease: a rationale based on homology between cerebral and retinal microvasculatures. J Anat. 2005;206(4):319-48. https://doi.org/10.1111/j. 1469-7580.2005.00395.x.

21. Ikram MK, Cheung CY, Wong TY, Chen CPLH. Retinal pathology as biomarker for cognitive impairment and Alzheimer's disease. J Neurol Neurosurg Psychiatry. 2012;83(9):917-22. https://doi.org/10.1136/ jnnp-2011-301628.

22. Campbell JP, Zhang M, Hwang TS, et al. Detailed vascular anatomy of the human retina by projection-resolved optical coherence tomography angiography. Sci Rep. 2017;7(January):1-11. https://doi.org/10.1038/ srep42201.

23. Joutel A, Monet-Leprêtre M, Gosel C, et al. Cerebrovascular dysfunction and microcirculation rarefaction precede white matter lesions in a mouse genetic model of cerebral ischemic smallvesseldisease. J Clin Invest. 2010;120(2):433-45

24. Fazekas F, Schmidt R, Scheltens P. Pathophysiologic mechanisms in the development of age-related white matter changes of the brain. Dement Geriatr Cogn Disord. 1998;9(SUPPL. 1):2-5. https://doi.org/10.1159/00005 1182

25. Yu D-Y, Cringle SJS. Intraretinal oxygen distribution in the monkey retina and the response to systemic hyperoxia. Invest Ophthalmol Vis Sci. 2005:46:4728-4733.

26. Cringle SJ, Yu D-Y, Paula KY, Su E-N. Intraretinal oxygen consumption in the rat in vivo. Invest Ophthalmol Vis Sci. 2002;43:1922-7.

27. Hagag AM, Pechauer AD, Liu L, et al. In response to Hyperoxia. Ophthalmol Retin. 2018;2(4):329-36. https://doi.org/10.1016/j.oret.2017.07.022. OCT.

28. Shahlaee A, Pefkianaki M, Hsu J, Ho AC. Measurement of Foveal Avascular Zone Dimensions and its Reliability in Healthy Eyes Using Optical Coherence Tomography Angiography. Am J Ophthalmol. 2016;161:50-5.e1. https://doi.org/10.1016/j.ajo.2015.09.026.

29. lafe NA, Phasukkijwatana N, Chen X, et al. Retinal Capillary Density and Foveal Avascular Zone Area Are Age-Dependent: Quantitative Analysis Using Optical Coherence Tomography Angiography. Invest Ophthalmol Vis Sci. 2016:57:5780-5787.

30. Fazekas F, Kleinert R, Offenbacher H, Schmidt R, Kleinert G, Payer F, et al. Pathologic correlates of incidental MRI white matter signal hyperintensities. Neurology. 1993;43:1683-9.
31. Ma KC, Ollson Y. The role of chronic brain edema in the formation of lacunes in Binswan- ger's encephalopathy. Cerebrovasc Dis. 1997;7:324-31.

32. Wen $C$, Pei $C$, Xu X, Lei J. Influence of axial length on Parafoveal and Peripapillary metrics from swept source optical coherence tomography angiography. Curr Eye Res. 2019;44(9):980-6. https://doi.org/10.1080/ 02713683.2019 .1607393$.

33. Coscas F, Sellam A, Glacet-Bernard A, Jung C, Goudot M, Miere ASE. Normative data for vascular density in superficial and deep capillary plexuses of healthy adults assessed by optical coherence tomography angiography. Invest Ophthalmol Vis Sci. 2016;57(9):OCT2.

34. Milani P, Montesano G, Rossetti L, Bergamini F, Pece A. Vessel density, retinal thickness, and choriocapillaris vascular flow in myopic eyes on OCT angiography. Graefes Arch Clin Exp Ophthalmol. 2018;256(8):1419-27. https://doi.org/10.1007/s00417-018-4012-y.

35. Tan CS, Lim LW, Chow VS, Chay IW, Tan S, Cheong KX, et al. Optical Coherence Tomography Angiography Evaluation of the Parafoveal Vasculature and Its Relationship With Ocular Factors. Invest Ophthalmol Vis Sci. 2016:57(9):OCT224-OCT234.

\section{Publisher's Note}

Springer Nature remains neutral with regard to jurisdictional claims in published maps and institutional affiliations.

Ready to submit your research? Choose BMC and benefit from:

- fast, convenient online submission

- thorough peer review by experienced researchers in your field

- rapid publication on acceptance

- support for research data, including large and complex data types

- gold Open Access which fosters wider collaboration and increased citations

- maximum visibility for your research: over $100 \mathrm{M}$ website views per year

At BMC, research is always in progress.

Learn more biomedcentral.com/submissions 